\title{
The role of diet and antioxidants in the prevention of Alzheimer's disease
}

\author{
Robert Dudkowiak', Anna Gryglas²,3, Elżbieta Poniewierka' \\ ${ }^{1}$ Division of Dietetics, Department of Gastroenterology and Hepatology, Wroclaw Medical University, Poland \\ ${ }^{2}$ Department of Neurology, Gromkowski Hospital, Wroclaw, Poland \\ ${ }^{3}$ Department of Social Pediatrics, Wroclaw Medical University, Poland
}

\begin{abstract}
Alzheimer's disease (AD) is the most common form of dementia among elderly. It is a progressive, neurodegenerative disorder of the brain which leads to the deterioration of cognitive, behavioral and impaired daily functioning and causes the gradual loss of independence. A significant portion of risk for dementia in old age is associated with lifestyle. Three important protective factors are diet, which should be rich in antioxidants, exercise and good cardiovascular health. It is believed that Mediterranean diet has a protective effect from dementia. This diet, rich in fruit and vegetables, legumes, olive oil, whole wheat bread, fish and seafood, with reduced consumption of red meat is also protective from cardiovascular diseases and promotes a healthy long life. There were some studies on the etiology of AD which noted an important role of vitamin B6, B12 and folic acid. All of them are involved in the metabolism of homocysteine, which is regarded as an independent risk factor for the development of $A D$, atherosclerosis and thrombosis. We also know that supplementation of vitamins $C$ and $E$ in the diet can be protective from AD. On the other hand we know that obesity and undernutrition can increase the risk of development of $A D$. As we can observe the aging of population we should remember that nutrition constitutes an interesting approach for the prevention of age-related brain disorders.
\end{abstract}

Keywords: Alzheimer's disease, reactive oxygen species, nutrition.

\section{Introduction}

Alzheimer's disease (AD) is progressive, neurodegenerative disorder of the brain which leads to the deterioration of cognitive, behavioral and impaired daily functioning and causes the gradual loss of independence. Multi-infarct dementia (vascular dementia), Parkinson's disease, Lewy body disease, Creutzfeldt-Jakob disease, Pick's disease, Huntington's disease are less common forms of dementia among elderly [1]. AD currently affects about $2 \%$ of the population in developed countries and its incidence is forecasted to increase significantly with the aging of the population [2]. US Census Bureau data predict that the number of people over 65 years amounting to about 35 million in 2000, will increase to 70 million in 2050, while the population of people over 85 years old will increase from 4.2 million in 2000 to 21 million in 2050 [3]. AD is the leading form of dementia in North America and Europe and it constitutes the significant part of care cost in these countries. It is considered as a global public health priority $[4,5]$. Etiology of AD is multi-factorial and it compounds of genetic and environmental factors including diet, physical activity, smoking and alcohol abuse. Barnes and Yaffe suggested that diabetes mellitus, midlife hypertension, midlife obesity, smoking, depression, and cognitive and physical inactivity are the main modifiable risk factors and represent around $50 \%$ of all cases of AD [6]. Diabetes, hypertension, and obesity are the civilization diseases where proper diet and physical activity are important elements of prevention and 
treatment. Dietary intake and nutritional state appear to be environmental lifestyle- related factors that might contribute to pathogenesis and slowing down AD [7-9]. Genetic factors are important especially in the generally accepted division of Alzheimer's disease into two types: sporadic AD and family AD [10]. Sporadic $A D$ also known as late-onset $A D$ constitutes more than $90 \%$ of all cases and occurs in patients aged 65 years old and older. The risk increases having the APOE $\varepsilon 4$ allele of apolipoprotein E (APOE) genotype [11]. Family $A D$ is more sporadic form of early onset $A D$ as its symptoms occur in younger age. Genetic variants of the amyloid precursor protein (APP) gene, presenilin 1 (PSEN 1) gene and presenilin 2 (PSEN 2) gene have been suggested to be associated with family AD [12].

\section{Alzheimer Disease and oxidative damage}

Amyloid $B(A ß)$ and tau are two main proteins playing an important role in pathogenesis of AD [13]. Formation of neuritic plaques composed of insoluble form of $A B$ in the brain and the accumulation of hyperphosphorylated form of tau in neurofibrillary tangles are the most important steps in the pathogenesis of $A D$ [14-16]. $A ß$ accumulation in the hippocampal region of the brain is thought to induce toxic effects, oxidative stress and immune response that lead to cognitive impairment [17]. The hyperphosphorylation of tau protein worsens the axonal transport and causes neuronal dysfunction in the central nervous system. Abnormal neurofibrillary tangles induce inflammatory response and activate cells (microglia, astrocytes, macrophages and lymphocytes) which causes exaggerated production of cytokines and chemokines [18]. Both, $A B$ and tau protein, induce inflammation in the vulnerable regions of the brain. This is considered to be an important factor in the onset of AD. The inflammatory process leads to neuronal dystrophy, overproduction of reactive oxygen species (ROS) and increased formation of $A B$ in the cerebral cortex and subcortical regions $[19,20]$. ROS are produced in the oxidative process which is the part of physiological reaction of the brain as a part of cellular signalling, metabolism and keeping the homeostasis [21, 22]. The lack of balance between the formation of free radicals and their removal is the cause of oxidative stress. Oxidative stress results in increased oxidation of lipids, proteins and nucleic acids which leads to cellular dysfunction. Chronic oxidative stress along with oxidative damage of the cerebral microvasculature and brain cells have become a potential pathogenesis of many neurodegenerative diseases such as age-related mild cognitive impairment, Parkinson's disease and Alzheimer's disease [23, 24]. Authors of the prospective study made in 2014 emphasize the increased oxidative stress in AD [25]. Exposing human body cells to chronic oxidative stress may exacerbate production of ROS and cause the death of cells including neurons. Amyloid $B$ can provoke oxidative stress by itself which is thought to be a crucial factor in the pathogenesis of $A D[26,27]$. The collection of $A B$ in the brain causes the dysfunction of mitochondria and metabolic disturbances as well as increased formation of the ROS [28]. Amyloid beta, as a derivative of transferrin, has strong affinity to transition metal ions such as iron, copper and zinc. It is able to reduce Fe3+ and Cu2+ producing ROS [29]. The human body has a mechanism to inactivate and detoxify ROS. The main antioxidants in the nervous system are superoxide dismutase, glutathione peroxidase and catalase. Some data show decreased levels of antioxidants in the plasma of patients with $A D$. The most important one is glutathione peroxidase which is a selenium-containing enzyme. Its activity in the blood is well correlated to the level of plasma selenium (Se) [30]. Pillai et al. have shown that Se deficiency is associated with cognitive decline, and that selenoproteins may inhibit the neurodegeneration in AD [31]. On the other hand Loef et al. concluded that there is no evidence for the role of Se in the treatment of AD [32]. However it still allows speculation on a potential preventive relevance. In a prospective study Krishnan and Rani noted that the blood Se may not be involved in regulating oxidative stress in AD. A longitudinal study which correlates plasma and cerebrospinal fluid Se and selenoprotein levels should be done [25]. The similar conclusions were reached by authors of systematic review and meta-analysis analyzing plasma nutrients among patients with AD [33]. The meta-analysis has shown significantly lower plasma levels of folate and vitamins A, B12, C and E in a group of AD patients compared to control population. There is a strong belief that vitamin $A$, vitamin $C$ and vitamin $E$ which are antioxidants may be beneficial in slowing the progression and preventing AD [34-36].

\section{Alzheimer Disease and diet}

Dietary intake and nutritional state are important environmental factors that affect the human body regardless of gender, age or comorbidity [37]. Proper nutrition is an important element of lifestyle, which plays 
a significant role in the human biological aging process. The quality of the food is also an important part of prevention and treatment of non-communicable, chronic metabolic diseases, the incidence of which increases with age [38]. Among these diseases is AD. They are included into neurodegenerative disorders.

An increasing number of publications emphasize the role of diet and dietary components in the development and treatment of dementia [39-41]. It is well known that nutritional deficiencies and a poorly balanced diet can lead to disturbances in the functioning of the body. Thus, some of the food components can serve a protective function, reducing the risk of developing diseases. Conducted epidemiological studies emphasize the role of the Mediterranean diet in reducing the risk of developing dementia and AD [42-44]. This diet, rich in fruit and vegetables, legumes, olive oil, whole wheat bread, fish and seafood, taking account of reduced consumption of red meat, is the source of all components of food. The importance of the individual components of the Mediterranean diet as a prevention of cognitive impairment is confirmed by numerous independent studies. Berr et al. conducted four-year follow-up and showed that a diet rich in olive oil reduces the risk of cognitive deficits among the elderly [45]. A prospective study in France over seven years and of 1416 people aged at least 68 years, assessed the effect of consumption of fish and meat on the risk of developing dementia [46]. Increased consumption of fish and seafood (at least 1 time per week) was associated with a significantly lower incidence of dementia. The benefits from consumption of fish was confirmed by the study made on a much larger population - 6158 people aged at least 65 years [47]. Hung et al. demonstrated that fatty fish consumption was associated with a lower risk of dementia and $A D$ in people without the APOE $\varepsilon 4$ [48]. During long-term follow-up, conducted among Swedish twins, it has been shown that fruit and vegetable intake was associated with a lower risk of developing dementia and $A D$ in women [49]. It has been demonstrated in a study of 193 healthy volunteers (aged 45 to 102 years) that regardless of gender, age, BMI and lipid parameters, people with a higher daily intake of fruit and vegetables performed better cognitive tests [50]. It has been stated that healthy subjects of any age with a high daily intake of fruit and vegetables have higher antioxidant levels, lower levels of biomarkers of oxidative stress, and better cognitive performance than healthy subjects of any age consuming low amounts of fruit and vegetables. Diet composition similar to the Medi- terranean diet seems to be the most appropriate and may exert a long-term beneficial effect on the functioning of the brain [51, 52]. This diet is worth recommendation as it lessens cognitive decline, reduces the progression of mild cognitive impairment to $A D$ and the overall risk of developing $A D$, and decreases mortality among patients with AD. Thus, high consumption of fish, olive oil, vegetables and fruit with a low glycemic index, seeds, beans, moderate consumption of wine and diary products such as cheese or yogurt, and low consumption of red meat and products without additional sugar is recommended. This diet is also recommended to reduce the risk of cardiovascular diseases, obesity, diabetes and hypertension. Therefore it seems to be a good way not only to prevent dementia but also to stay in a better health [53].

The high content of antioxidants in fruit and vegetables is one of the advantages of the Mediterranean diet. Omega-3 acids ( $\alpha$-linolenic acids) and omega- 6 acids (linoleic acids) which belong to polyunsaturated fatty acids (PUFA) are equally important. It is also known that PUFA are precursors of leukotrienes, prostaglandins and cell membranes. Vegetable oils (sunflower, soybean, corn, rapeseed) and the acid of the omega-3 family - fish oil, linseed oil and walnuts are the source of linoleic acids in the human diet.

The importance of fish and seafood consumption in the prevention of $A D$ was punctuated by Barberger-Gateau et al. [46] and by the authors of Rotterdam Study [54]. Kalmijn et al. found that a high intake of total fat, saturated fat and cholesterol was associated with an increased risk of dementia. On the other hand they have assessed that the diet rich in fish reduced the risk of $A D$.

Increased content of omega-3 in a serum and in a diet was reducing the risk of dementia which was noted in few studies [55-57]. Some authors say that there is no significant correlation between PUFA intake and the risk of dementia, including $A D$ [58-60]. Therefore the importance of fats, especially PUFA, in the etiopathogenesis of $A D$ requires further investigation.

\section{Alzheimer Disease and vitamins}

Healthy eating and proper diet will ensure proper nutrition regardless of age and provide the body with all the essential nutrients, including micro- and macronutrients. Please note that in the elderly, we often deal with comorbidities, and above all the involuntary changes in the process of digestion and absorption. The unbalanced diet and increased demand for 
protein and vitamins in the elderly is caused by the decreased absorption from the digestive tract which causes significantly reduced health [61]. It is commonly known that the brain is susceptible to oxidative stress and damage as a result of its high metabolic rate and relatively lower regenerative capacity. The brain uses approximately $20 \%$ of total body oxygen consumption. A number of publications emphasize the role of both, the antioxidant vitamins ( $E, C$, carotenoids including vitamin A) and vitamins involved in the metabolism of homocysteine (vitamin B6, B12 and folic acid). An excessive amount of ROS, in addition to the development of the inflammatory processes, also contributes to faster aging. The neural tissue manifests large oxygen consumption, increased mitochondria density and a high content of polyunsaturated fatty acids in the cell membranes. This causes elevated sensitiveness to harmful action of the free radicals and peroxides [62]. The significant role in the prevention of this damage plays vitamin E (tocopherol). In the 90s a group of scientists from Australia, showed a relationship between the concentration of vitamin $E$ in the serum and cognitive function among people over 60 years of age. Their studies have been conducted to increase the prevention of stroke [63]. Perkins et al. reported a relationship between low serum level of vitamin $E$ and cognitive impairment [64]. In study, which was carried out among 4809 participants showed that there had been no correlation between the level of vitamin C, carotenoids including vitamin A and cognitive function. Also, there was a study conducted in a group of 442 people which showed the reversed result - ascorbic acid, carotenoids, and their plasma concentration only were associated with a better performance of memory [65]. The protective role of the products containing vitamin $E$ in diet was observed in seven-year prospective study made among 815 healthy people over 65 years of age. They found it as a preventing factor against the development of $A D$ [66]. Ortega et al. emphasized the importance of an adequate supply of vitamin $\mathrm{E}$ in the diet. They made an analysis of the diet of elderly people for 5 days [67]. The smaller intake of vitamin $\mathrm{E}$ in the diet and its lower concentration in the serum was associated with worse outcomes of the cognitive tests. It is punctuated that not only the alpha-tocopherol, but different forms of vitamin $\mathrm{E}$ as well, play an important role in the prevention of $A D$ [68]. Vitamin $E$ which comes from dietary supplements has not been shown to reduce Alzheimer's disease risk. Many common supplements provide alpha-tocopherol only. Most of them do not replicate the range of forms of vitamin $E$ that we find in regular food. It has been shown that high intake of alpha-tocopherol reduces serum concentrations of gamma- and delta-tocopherols [69]. It could be the explanation why the higher intake of vitamin $\mathrm{E}$ with food was associated with reduced Alzheimer's disease incidence in the study made by Morris et al. [66]. Similarly, in the Rotterdam Study, high vitamin E intake from natural sources was associated with reduced dementia incidence [70]. There was also a prospective study of 4,740 people over 65 years old, that has shown that supplementation of vitamin $E$ and $C$ was associated with a reduction in the incidence of AD [71]. Equally, another study made among 5395 participants, aged over 55, showed that a high intake of vitamin $E$ and $C$ was associated with a lower risk of AD. This association was noticed regardless of the level of education and the presence of APOE $\varepsilon 4$ [72]. However, the reports describing the role of other antioxidative vitamins are divergent. In a large prospective cohort study made by Devore at al., intake of vitamin C, carotenoids and flavonoids had no effect on the risk of dementia including $A D$ [70].

Studies on the role of diet in pathogenesis and course of AD underlined an influence of vitamins B6, B12 and folic acid. These vitamins are involved in the metabolism of homocysteine, which is in the human body as an intermediate product formed from exogenous, derived from protein intake methionine on the way to endogenous cysteine. Homocysteine is regarded as an independent risk factor for atherosclerosis and thrombosis. High blood levels of homocysteine (hyperhomocysteinemia) are also a risk factor for the development of cognitive disorders and dementia including $A D$ $[73,74]$. Elevated level of homocysteine and decreased level of vitamin B12 in the plasma of patients with AD was demonstrated by Malaguarnera et al. [75]. In a nearly 10 years study conducted among older people without dementia we had observed that folate intake was associated with a lower risk of developing $A D$ [76]. This dependency was not observed for vitamin B12, vitamin C and carotenoids. There was a publication punctuating that serum levels of vitamin B12 and folic acid may be important in preventing AD [77]. In another study of 370 people without senile dementia over the age of 70 it has been observed that low levels of vitamin B12 and folic acid in their blood correlated with an increased risk of developing AD. The level of folic acid below $10 \mathrm{pmol} / \mathrm{L}$ and vitamin B12 below $150 \mathrm{pmol} / \mathrm{L}$ doubled the risk of dementia. Walker et al. demonstrated that long-term supplementation with folic acid at a daily dose of $400 \mathrm{mg}$ and vitamin B12 at dose of $100 \mathrm{mg}$, improved cognitive function. It was 
made on the basis of two years of observation [78]. On the other hand we have also found reports of large prospective studies with no association between the consumption of vitamin B6, vitamin B12 and folic acid and the risk of $A D[79,80]$. We also know that elevated levels of homocysteine are neurotoxic and linked with cardiovascular dysfunction, cognitive decline, increased risk of dementia and brain atrophy [81].

The role of vitamins in the prevention and course of $A D$ is not clear. There is still a need to make further research where several other dependencies should be considered like comorbidities or the way of supplementation of vitamins.

\section{Conclusion}

$A D$ is the most common form of dementia and affects about $2 \%$ of the population in developed countries. It will still increase with the aging of the population. Therefore, effective prevention and alleviation of symptoms associated with AD is so important. The proceedings should aim to delay the time of development and reduce the prevalence of cognitive impairment which would allow elderly to live longer and independently. Currently available medications may reduce the symptoms of $A D$ and slow down the progression of the disease however these do not lead to a complete cure. It explains why the knowledge of risk factors of AD should become important part of proceedings. We know that diabetes mellitus, midlife hypertension and obesity, smoking, depression and cognitive and physical inactivity are the main modifiable risk factors which represent about $50 \%$ of AD cases. Lifestyle, including proper nutrition and proper weight are important factors in prevention of diseases mentioned before. We also know that diet is a part of non-pharmacological prevention of cardiovascular diseases. The Mediterranean diet improves the health and reduces the risk of many diseases including AD. The supplementation of antioxidative vitamins, such a vitamin $C$ and $E$, seems to reduce the risk of $A D$. What we should remember is that malnutrition accompanies $A D$ quite often. There has been some studies saying that accelerated loss of weight may precede the diagnosis of $\operatorname{AD}[82,83]$. The importance of the single components of the diet, like berries, has not been documented well and requires further analysis [84].

Nevertheless the components of diet play an important role in reducing oxidative stress, modulating the immune response, reducing inflammation process and in providing elements for the body construction.
Following that we may understand why the diet is so important for proper brain function and that it may be protective from AD. However, there are only few randomized clinical trials that have been designed to test the role of diet in cognitive decline and in dementia including AD. We still need more studies to prepare the multi-nutrient strategy for people with dementia and to unravel the specific influence of each dietary component on cognitive functions. We should be aware of synergistic interactions between different nutrients and we should keep in mind that there is a positive impact of antioxidants on our brain. We should also remember that connecting medications with non- pharmacological treatment like appropriate diet, physical and mental activity may improve the overall functioning of patients with dementia.

\section{Acknowledgements}

\section{Conflict of interest statement}

The authors declare no conflict of interest.

\section{Funding sources}

There are no sources of funding to declare.

\section{References}

1. Dickson DW. Neuropathology of Alzheimer's disease and other dementias. Clin Geriatric Med. 2001;17:209-228.

2. Hebert LE, Weuve J, Scherr PA, Evans DA. Alzheimer disease in the United States (2010-2050) estimated using the 2010 census. Neurology. 2013. 80:1778-1783.

3. Neugroschl J, Sano M. An update on treatment and prevention strategies for Alzheimer's disease. Curr. Neurol. Neurosci. Rep. 2009;9:368-376.

4. Hurd MD, Martorell P, Delavande A, Mullen KJ, Langa KM. Monetary costs of dementia in the United States. N Engl J Med. 2013;368:1326-1334.

5. World Health Organization. Dementia: a public health priority. WHO Geneva. 2013.

6. Barnes DE, Yaffe K. The projected effect of risk factor reduction on Alzheimer's disease prevalence. Lancet Neurol. 2013;10:819-828.

7. Sezgin Z, Dincer Y. Alzheimer's disease and epigenetic diet. Neurochem Int. 2014;78:105-116.

8. Shah R. The role of nutrition and diet in Alzheimer disease: a systematic review. J Am Med Dir Assoc. 2013;14:398402.

9. Ogawa S. Nutritional management of older adults with cognitive decline and dementia. Geriatr Gerontol Int. 2014;14 Suppl 2:17-22.

10. Blennow K, de Leon MJ, Zetterberg H. Alzheimer's disease. Lancet 2006;368:387-403.

11. Guerreiro R, Bras J, Toombs J, Heslegrave A, Hardy J, Zetterberg $\mathrm{H}$. Genetic variants and related biomarkers in sporadic Alzheimer's disease. Curr Genet Med Rep. 2015;3:19-25.

12. Zou Z, Liu C, Che C, Huang H. Clinical genetics of Alzheimer's disease. Biomed Res Int. 2014;2014:291862. 
13. Ittner LM, Götz J. Amyloid- $\beta$ and tau - a toxic pas de deux in Alzheimer's disease. Nat Rev Neurosci. 2011;12:65-72.

14. Huang HC, Jiang ZF. Accumulated amyloid-beta peptide and hyperphosphorylated tau protein: relationship and links in Alzheimer's disease. J Alzheimers Dis. 2009;16:1527.

15. Brunden KR, Ballatore $C$, Crowe $A$, Smith $A B$ 3rd, Lee VM, Trojanowski JQ. Tau-directed drug discovery for Alzheimer's disease and related tauopathies: a focus on tau assembly inhibitors. Exp Neurol. 2010;223:304-10.

16. Dickson DW. Neuropathological diagnosis of Alzheimer's disease: a perspective from longitudinal clinicopathological studies. Neurobiol Aging. 1997;18: S21-6.

17. Anantharaman M, Tangpong J, Keller JN, Murphy MP, Markesbery WR, Kiningham KK, et al. Beta-amyloid mediated nitration of manganese superoxide dismutase: implication for oxidative stress in a APPNLH/NLH X PS-1P264L/P264L double knock-in mouse model of Alzheimer's disease. Am J Pathol. 2006;168:1608-18.

18. Wang DB1, Dayton RD, Zweig RM, Klein RL. Transcriptome analysis of a tau overexpression model in rats implicates an early pro-inflammatory response. Exp Neurol. 2010;224:197-206.

19. Fassbender K, Masters C, Beyreuther K. Alzheimer's disease: an inflammatory disease? Neurobiol Aging. 2000;21:433-6, discussion 451-3.

20. Hill JM, Dua P, Clement C, Lukiw WJ. An evaluation of progressive amyloidogenic and pro-inflammatory change in the primary visual cortex and retina in Alzheimer's disease (AD). Front Neurosci. 2014;12:8:347.

21. Cahill-Smith S, Li JM. Oxidative stress, redox signalling and endothelial dysfunction in ageing-related neurodegenerative diseases: a role of NADPH oxidase 2. Br J Clin Pharmacol. 2014;78:441-53.

22. Sochocka M, Koutsouraki ES, Gasiorowski K, Leszek J. Vascular oxidative stress and mitochondrial failure in the pathobiology of Alzheimer's disease: a new approach to therapy. CNS Neurol Disord Drug Targets. 2013;12:87081.

23. Schrag M, Mueller C, Zabel M, Crofton A, Kirsch WM, Ghribi O, et al. Oxidative stress in blood in Alzheimer's disease and mild cognitive impairment: a meta-analysis. Neurobiol Dis. 2013;59:100-10.

24. Leszek J, Sochocka M, Gasiorowski K. Vascular factors and epigenetic modifications in the pathogenesis of Alzheimer's disease. J Neurol Sci. 2012;323:25-32.

25. Krishnan S, Rani P. Evaluation of selenium, redox status and their association with plasma amyloid/tau in Alzheimer's disease. Biol Trace Elem Res. 2014;158:15865.

26. Carrano A, Hoozemans JJM, van der Vies SM, Rozemuller AJM, van Horssen J, de Vries HE. Amyloid Beta induces oxidative stress-mediated blood-brain barrier changes in capillary amyloid angiopathy. Antioxid Redox Signal. 2011;15:1167-78.

27. Swomley AM, Förster S, Keeney JT, Triplett J, Zhang Z, Sultana $\mathrm{R}$, et al. Abeta, oxidative stress in Alzheimer disease: evidence based on proteomics studies. Biochim Biophys Acta. 2014;1842:1248-57.

28. Sheng B, Wang X, Su B, Lee HG, Casadesus G, Perry G, Zhu X. Impaired mitochondrial biogenesis contributes to mitochondrial dysfunction in Alzheimer's disease. J Neurochem. 2012;120:419-29.

29. Finefrock AE, Bush Al, Doraiswamy PM. Current status of metals as therapeutic targets in Alzheimer's disease. J Am Geriatr Soc. 2003;51:1143-8.

30. Puertas MC, Martínez-Martos JM, Cobo MP, Carrera MP, Mayas MD, Ramírez-Expósito MJ. Plasma oxidative stress parameters in men and women with early stage Alzheimer type dementia. Exp Gerontol. 2012;47:625-30.

31. Pillai R, Uyehara-Lock JH, Bellinger FP. Selenium and selenoprotein function in brain disorders. IUBMB Life. 2014;66:229-39.

32. Loef M, Schrauzer GN, Walach H. Selenium and Alzheimer's disease: a systematic review. J Alzheimers Dis. 2011;26:81-104.

33. Lopes da Silva S, Vellas B, Elemans S, Luchsinger J, Kamphuis P, Yaffe K, et al. Plasma nutrient status of patients with Alzheimer's disease: Systematic review and meta-analysis. Alzheimers Dement. 2014;10:485-502.

34. Di Domenico F, Barone E, Perluigi M, Butterfield DA. Strategy to reduce free radical species in Alzheimer's disease: an update of selected antioxidants. Expert Rev Neurother. 2015;15:19-40.

35. Ramesh BN, Rao TS, Prakasam A, Sambamurti K, Rao KS. Neuronutrition and Alzheimer's disease. J Alzheimers Dis. 2010;19:1123-1139.

36. Morris MC. The role of nutrition in Alzheimer's disease: Epidemiological evidence. Eur J Neurol. 2009;16:1-7.

37. Esmailnasab N, Moradi G, Delaveri A. Risk Factors of Non-Communicable Diseases and Metabolic Syndrome. Iran J Public Health. 2012;41:77-85.

38. Solfrizzi V, Panza F, Frisardi V, Seripa D, Logroscino G, Imbimbo BP, Ilotto A. Diet and Alzheimer's disease risk factors or prevention: the current evidence. Expert Rev Neurother. 2011;11:677-708.

39. Shatenstein B, Kergoat MJ, Reid I, Chicoine ME. Dietary intervention in older adults with early-stage Alzheimer dementia: early lessons learned. J Nutr Health Aging. 2008;12:461-469.

40. Salva A, Andrieu S, Fernandez, Moulin J, Decarli B, Rojano-i-Luque $X$, et al. Health and nutritional promotion program for patients with dementia (NutriAlz Study): design and baseline data. J Nutr Health Aging. 2009;13:529537.

41. Wyka J. Nutritional factors in prevention of Alzheimer's disease. Rocz Panstw Zakl Hig. 2012;63:135-40.

42. Scarmeas N, Stern Y, Mayeux R, Manly JJ, Schupf N, Luchsinger JA. Mediterranean diet and mild cognitive impairment. Arch Neurol. 2009;66:216-225.

43. Knopman D. Mediterranean diet and late-life cognitive impairment: a taste of benefit. JAMA. 2009;302:686687.

44. Feart C, Samieri C, Alle`s B, Barberger-Gateau P. Potential benefits of adherence to the Mediterranean diet on cognitive health. Proc Nutr Soc. 2013;72:140-152.

45. Berr C, Portet F, Carriere I, Akbaraly TN, Feart C, Gourlet $V$, et al. Olive oil and cognition: Results from the threecity study. Dement Geriatr Cogn Disord. 2009;28:357-364.

46. Barberger-Gateau P, Letenneur L, Deschamps V, Pérès K, Dartigues JF, Renaud S. Fish, meat, and risk of dementia: Cohort study. BMJ 2002;325:932-933. 
47. Morris M, Evans D, Tangney C, Bienias JL, Wilson RS. Fish consumption and cognitive decline with age in a large community study. Arch Neurol. 2005;62:1849-1853.

48. Huang T, Zandi P, Tucker K, Fitzpatrick AL, Kuller $L H$, Fried $L P$, et al. Benefits of fatty fish on dementia risk are stronger for those without APOE epsilon4. Neurology. 2005;65:1409-1414.

49. Hughes $T$, Andel R, Small B, Borenstein AR, Mortimer $J A$, Wolk $A$, et al. Midlife fruit and vegetable consumption and risk of dementia in later life in Swedish twins. Am J Geriatr Psychiatry. 2010;18:413-420.

50. Polidori M, Praticó D, Mangialasche F, Mariani E, Aust $\mathrm{O}$, Anlasik T, et al. High Fruit and Vegetable Intake is Positively Correlated with Antioxidant Status and Cognitive Performance in Healthy Subjects. J Alzheimers Dis. 2009;17:921-927.

51. Lourida I, Soni M, Thompson-Coon J, Purandare N, Lang IA, Ukoumunne OC, et al. Mediterranean diet, cognitive function, and dementia: a systematic review. Epidemiology. 2013;24:479-489.

52. Feart C, Samieri C, Barberger-Gateau P. Mediterranean diet and cognitive health: an update of available knowledge.Curr Opin Clin Nutr Metab Care. 2015;18:51-62.

53. Deckers K1, van Boxtel MP, Schiepers OJ, de Vugt M, Muñoz Sánchez JL, Anstey KJ, et al. Target risk factors for dementia prevention: a systematic review and Delphi consensus study on the evidence from observational studies. Int J Geriatr Psychiatry. 2015;30:234-46.

54. Kalmijn S, Launer L, Ott A. Dietary fat intake and the risk of incydent dementia in the Rotterdam Study. Ann Neurol. 1997;42:776-782.

55. Lopez L, Kritz-Silverstein D, Barrett Connor E. High dietary and plasma levels of the omega-3 fatty acid docosahexaenoic acid are associated with decreased dementia risk: the Rancho Bernardo study. J Nutr Health Aging. 2011;15:25-31.

56. Naqvi AZ, Harty B, Mukamal KJ, Stoddard AM, Vitolins M, Dunn JE. Monounsaturated, trans, and saturated Fatty acids and cognitive decline in women. J Am Geriatr Soc. 2011;59:837-843.

57. Samieri C, Feart C, Proust-Lima C, Peuchant E, Dartigues JF, Amieva $\mathrm{H}$, et al. Omega-3 fatty acids and cognitive decline: modulation by ApoEepsilon4 allele and depression. Neurobiol Aging. 2011;32:2317-13-22.

58. Devore EE, Grodstein F, van Rooij FJ, Hofman A, Rosner B, Stampfer MJ, et al. Dietary intake of fish and omega-3 fatty acids in relation to long-term dementia risk. Am J Clin Nutr. 2009;90:170-176.

59. Kröger E, Verreault R, Carmichael P, Lindsay J, Julien $P$, Dewailly $E$, et al. Omega-3 fatty acids and risk of dementia: The Canadian Study of Health and Aging. Am J Clin Nutr. 2009;90:184-192.

60. Sydenham E, Dangour AD, Lim WS. Omega 3 fatty acid for the prevention of cognitive decline and dementia. Cochrane Database Syst Rev. 2012 6: CD005379.

61. Marshall T, Stumbo P, Warren J, Xie X-J. Inadequate nutrient intakes are common and are associated with low diet variety in rural, community-dwelling elderly. J. Nutr. 2001;131:2192-2196.

62. Selvatici R, Marani L, Marino S, Siniscalchi A. In vitro mitochondrial failure and oxidative stress mimic bio- chemical features of Alzheimer disease. Neurochem Int. 2013;63:112-1120.

63. Schmidt R, Hayn M, Reinhard B. Plasma antioxidants and cognitive performance in middle-aged and older adults: results of the Austrian Stroke Prevention Study. J Am Geriatr Soc. 1998;46:1407-1410.

64. Perkins A, Hendrie H, Callahan C. Association of antioxidants with memory in a multiethnic elderly sample using the Third National Health and Nutrition Examination Survey. Am J Epidemiol. 1999;150:37-44.

65. Perrig W, Perrig P, Stahelin H. The relation between antioxidants and memory performance in the old and very old. J Am Geriatr Soc. 1997;45:718-724.

66. Morris M, Evans D, Bienias J. Dietary intake of antioxidant nutrients and the risk of incident Alzheimer diseases in a Biracial Community Study. JAMA. 2002;287:32303237.

67. Otrega R, Requejo A, Lopez-Sobaler A, Andres P. Cognitive Function in Elderly People is influence by vitamin E status. J Nutr. 2002;132:2065-2068.

68. Morris M, Evans DA, Tangney CC, Bienias JL, Wilson RS, Aggarwal NT, et al. Relation of the tocopherol forms to incident Alzheimer disease and to cognitive change. Am J Clin Nutr. 2005;81:508-514.

69. Huang HY, Appel LJ. Supplementation of diets with alpha-tocopherol reduces serum concentrations of gamma- and delta-tocopherol in humans. J Nutr. 2003;133:3137-40.

70. Devore EE, Grodstein F, van Rooij FJ, Hofman A, Stampfer MJ, Witteman JC, et al. Dietary antioxidants and long-term risk of dementia. Arch Neurol. 2010;67:819-25.

71. Zandi P, Anthony J, Khachaturian A, Stone S. Reduced risk of Alzheimer disease in users of antioxidant vitamin supplements. Arch. Neurol. 2004;61:82-88.

72. Engelhart $M$, Geerlings $M$, Ruitenberg $A$, van Swieten JC, Hofman A, Witteman JC, et al.. Dietary intake of antioxidants and risk of Alzheimer disease. JAMA. 2002;287:3223-3229.

73. Morris MS, Jacques PF, Rosenberg IH, Selhub J. Hyperhomocysteinemia associated with poor recall in the third national health and nutrition examination survey. Am J Clin Nutr. 2001;73:927-933.

74. Lehmann M, Gottfries CG, Regland B. Identification of cognitive impairment in the elderly: homocysteine is an early marker. Dement Geriatr Cogn. 1999;10:12-20.

75. Malaguarnera M, Ferri R, Bella R. Homocysteine, vitamin B12 and folate in vascular dementia and in Alzheimer disease. Clin Chem Lab Med. 2004;42:1032-1035.

76. Corrada M, Kawas C, Hallfrisch J, Muller D, Brookmeyer R. Reduced risk of alzheimer's disease with high folate intake: The Baltimore Longitudinal Study of Aging. Alzheimer's \& Dement. 2005;1:11-18.

77. Wang $\mathrm{H}$, Wahlin A, Basun H. Vitamin B12 and foliate in relation to the development of Alzheimer 's disease. Neurology. 2001;56:1188-1194.

78. Walker J, Batterham P, MacKinnon A, Jorm AF, Hickie I, Fenech $M$, et al. Oral folic acid and vitamin B-12 supplementation to prevent cognitive decline in community-dwelling older adults with depressive symptoms - the Beyond Ageing Project: A randomized controlled trial. Am J Clin Nutr. 2012;95:194-203.

79. Morris M, Evans D, Schneider J, Tangney CC, Bienias JL, Aggarwal NT. Dietary folate and vitamins B-12 and 
B-6 not associated with incident Alzheimer's disease. J Alzheimers Dis. 2006;9:435-443.

80. Nelson C, Wengreen H, Munger R, Corcoran C. Dietary folate, vitamin B-12, vitamin B-6 and incident Alzheimer's disease: The cache county memory, health and aging study. J Nutr Health Aging. 2009;13:899-905.

81. Madsen SK, Rajagopalan P, Joshi SH, Toga AW, Thompson PM. Alzheimer's Disease Neuroimaging Initiative (ADNI). Higher homocysteine associated with thinner cortical gray matter in 803 participants from the Alzheimer's Disease Neuroimaging Initiative. Neurobiol Aging. 2015; 36: 203-210.
Correspondence address:

Robert Dudkowiak

Division of Dietetics

Department of Gastroenterology and Hepatology Wroclaw Medical University, Poland email: robindud@op.pl

Acceptance for editing: 2016-06-10

Acceptance for publication: 2016-06-23 